KIAS-P06003

hep-th/0603142

\title{
From Marginal Deformations to Confinement
}

\author{
Changhyun Ahn ${ }^{1}$ and Justin F. Vázquez-Poritz ${ }^{2}$ \\ 1 Department of Physics, Kyungpook National University, Taegu 702-701, Korea \\ 2 Department of Physics, University of Cincinnati, Cincinnati OH 45221-001, USA \\ ahn@knu.ac.kr, jporitz@physics.uc.edu
}

\begin{abstract}
We consider type IIB supergravity backgrounds which describe marginal deformations of the Coulomb branch of $\mathcal{N}=4$ super Yang-Mills theory with $S O(4) \times S O(2)$ global symmetry. Wilson loop calculations indicate that certain deformations enhance the Coulombic attraction between quarks and anti-quarks at the UV conformal fixed-point. In the IR region, these deformations can induce a transition to linear confinement.
\end{abstract}




\section{Introduction}

It has been conjectured that type IIB superstring theory on $A d S_{5} \times S^{5}$ is dual to fourdimensional $\mathcal{N}=4$ super Yang Mills theory [1, 2, 3]. The supergravity dual of marginal deformations of this theory related to the $U(1) \times U(1)$ global symmetry was found in [4]. This involved T-dualizing along a $U(1)$ direction of the 5-sphere, lifting to eleven dimensions and then going back to type IIB theory along $U(1)$ directions which are shifted by an $S L(3, R)$ transformation [4]. So long as the direction corresponding to the $U(1) \mathrm{R}$-symmetry of the theory is not involved in this procedure, the deformed theory preserves $\mathcal{N}=1$ supersymmetry. Matching this deformation of $A d S_{5} \times S^{5}$ to an exactly marginal operator in the field theory provides a holographic test of the methods in [5]. In this manner, marginal deformations have been found for various superconformal field theories, including those associated with the conifold as well as the $Y^{p, q}$ spaces [4] and the $L^{p, q, r}$ spaces [6] ${ }^{1}$. Also, the eleven-dimensional supergravity duals of marginal deformations of various three-dimensional conformal field theories were found in [6, 11].

This method can also be used to find the gravity duals of field theories undergoing renormalization group (RG) flows 4, 12, 13. In particular, the gravity dual description of deformations of the Coulomb branch of $\mathcal{N}=4$ Yang Mills theory with $S O(2)^{3}$ global symmetry was considered in [13]. This part of the moduli space corresponds to a continuous distribution of D3-branes on an ellipsoidal shell [14. The introduction of scalar expectation values does not change the superpotential of the theory. Furthermore, the superpotential along the deformed Coulomb branch flow is the same as that for the marginal deformations at the UV conformal fixed point [4], and is given by

$$
W=\operatorname{Tr}\left(\Phi_{1} \Phi_{2} \Phi_{3}-q \Phi_{2} \Phi_{1} \Phi_{3}\right), \quad q^{n}=1
$$

which is a $q$-deformation of the superpotential preserving $\mathcal{N}=1$ supersymmetry [5]. $\Phi_{i}$ are three adjoint chiral superfields, $q=e^{2 \pi i\left(\gamma-\tau_{s} \sigma\right)}$ and $\tau_{s}$ is related to the gauge coupling and theta parameter of the field theory.

The setup can be pictured as in Figure 1. The $\mathcal{N}=4$ super Yang Mills theory undergoes the $\mathrm{RG}$ flow on the left when certain $S O(6)$ scalar fields acquire non-vanishing expectation values. We will focus on a part of the moduli space for which Wilson loop calculations [15, 16] indicate that there is a Coulombic force between quarks and anti-quarks [17]. After applying $\sigma$ deformations to the UV conformal fixed point, the theory undergoes a different RG flow, as shown on the right of Figure $1^{2}$. How do the marginal deformations of the UV conformal

\footnotetext{
${ }^{1}$ The Sasaki-Einstein spaces $Y^{p, q}$ and $L^{p, q, r}$ were found in [7, 8, and 9, 10, respectively.

${ }^{2}$ This picture is a bit over-simplified, since there is actually a countably infinite family of deformations
} 
fixed point effect the physics of the IR region? From the Wilson loops, we find that these $\sigma$ deformations can actually cause the theory to undergo a phase transition such that the quark anti-quark pairs exhibit linear confinement ${ }^{3}$. Similar phenomena were discussed on the gauge theory side in [19, 20, 21]. We should note that we only consider external quarks.

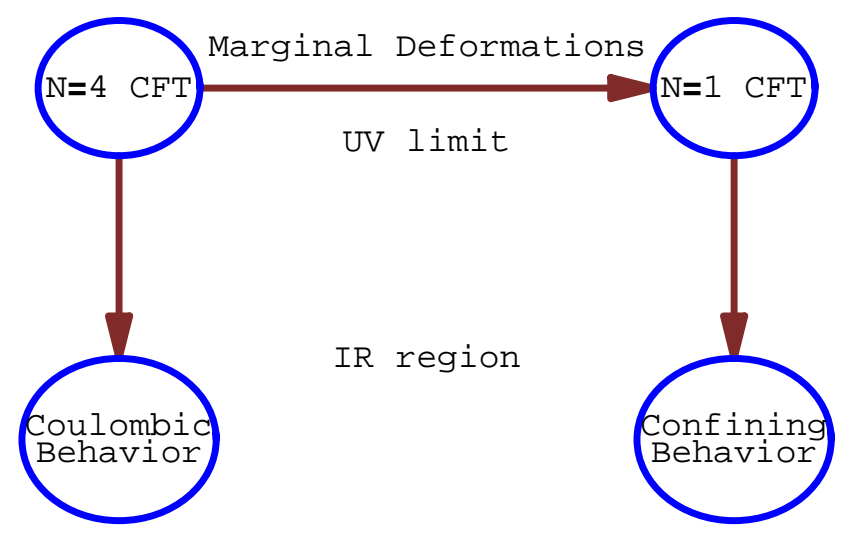

Figure 1: Marginally deformations can cause conformal field theories to undergo different Renormalization Group flows. The IR region of these theories can be drastically changed. We will consider examples in which $\sigma$ deformations of the UV conformal fixed point induces a transition in the IR region from Coulombic behavior to linear confinement.

This paper is organized as follows. In section 2, we show that the marginal deformations of $\mathcal{N}=4$ super Yang Mills theory enhance the Coulombic attraction between quarks and anti-quarks. In section 3, show that deformations along certain parts of the moduli space of the Coulomb branch cause a phase transition to a linearly confining theory. We discuss various issues and further directions in section 4 .

\section{Marginal deformations of $\mathbf{A d S}_{5} \times S^{5}$}

For the $\mathrm{AdS}_{5} \times S^{5}$ background of type IIB theory, Wilson loop calculations indicate that the energy of a string stretched along the radial direction is $E=c / L$, where $L$ is the distance between the string endpoints and the strength parameter $c$ is proportional to $\sqrt{g_{Y M}^{2} N}$ [16, 15]. This is to be expected from conformal invariance of the dual four-dimensional gauge theory

that can be applied at all points along the original RG flow.

${ }^{3}$ Wilson loops for string configurations which are not purely radially oriented have been studied for the $\gamma$ deformed backgrounds [18]. 
[1] ${ }^{4}$. The Lunin-Maldacena deformation of this background has the string-frame metric [4]

$$
d s_{s t r}^{2}=\alpha^{\prime} \sqrt{H}\left(\frac{r^{2}}{R^{2}} d x_{\mu}^{2}+R^{2} \frac{d r^{2}}{r^{2}}+R^{2} d s_{\tilde{S}^{5}}\right)
$$

where the characteristic length scale of $\mathrm{AdS}_{5}$ is given by $R^{4}=4 \pi g_{Y M}^{2} N$ in string units. $\tilde{S}^{5}$ is the deformation of $S^{5}$ which depends on the modulus of $\beta=\gamma-\tau_{s} \sigma$, where $\gamma$ and $\sigma$ are real deformation parameters and $\tau_{s}$ is a complex structure parameter related to the gauge coupling and theta parameter of the dual gauge theory [4. The conformal factor $H$ is given by

$$
H=1+4 \hat{\sigma}^{2} s_{\alpha}^{2}\left(c_{\alpha}^{2}+s_{\alpha}^{2} s_{\theta}^{2} c_{\theta}^{2}\right)
$$

where $\alpha$ and $\theta$ are internal coordinates of $\tilde{S}^{5}$ and $\hat{\gamma} \equiv \gamma R^{2} / 2, \hat{\sigma} \equiv \sigma R^{2} / 2$. The classical supergravity description is valid as long as the curvature is small relative to the string scale, the two-torus corresponding to the $U(1)^{2}$ global symmetry is larger than the string scale, and the metric does not degenerate at arbitrary points. These conditions can be met for [4]

$$
R>>1, \quad \hat{\gamma}<<R, \quad \hat{\sigma}<<R
$$

From the AdS/CFT correspondence, the gluonic field created by a static quark anti-quark pair can be described by a string configuration whose endpoints are located on the boundary of the AdS spacetime [15, 16. The Wilson loop can be computed by minimizing the NambuGoto action for a fundamental string on the supergravity background. The action for the Euclideanized string worldsheet is given by

$$
S=\frac{1}{2 \pi \alpha^{\prime}} \int d \tau d \sigma \sqrt{\operatorname{det} G_{M N} \partial_{\alpha} X^{M} \partial_{\beta} X^{N}}
$$

For a static configuration, we can take $\tau=t$ and $\sigma=x_{1}$. Also, because our configuration is static, the boundary action due to the antisymmetric tensor field vanishes does not contribute to the string action.

In order for a purely radial string to solve the equations of motion, it is required that $\partial_{\alpha} H=\partial_{\theta} H=0$. For example, $H=1+\hat{\sigma}^{2}$ for $\theta=0$ and $\alpha=\pi / 4$, as well as $\theta=\pi / 4$ and $\alpha=\pi / 2$. Also, $H=1+\frac{4}{3} \hat{\sigma}^{2}$ for $\theta=\pi / 4$ and $c_{\alpha}=1 / \sqrt{3}$. Then

$$
S=\frac{T \sqrt{H}}{2 \pi} \int d x_{1} \sqrt{\left(\partial_{x_{1}} r\right)^{2}+r^{4} / R^{4}} .
$$

\footnotetext{
${ }^{4}$ This carries over when $S^{5}$ is replaced by $T^{1,1}$ or one of the countably infinite $Y^{p, q}[\overline{7}]$ or $L^{p, q, r}$ spaces 9. 10.
} 
Since $H$ is a constant, the Wilson loop calculation proceeds as in [16] with the result that the energy of the string configuration is

$$
E=-\frac{4 \pi^{2} \sqrt{2 g_{Y M}^{2} N H}}{\Gamma(1 / 4)^{4} L},
$$

where $L$ is the distance between the string endpoints. We will show the intermediate steps of such computations in the next section, which includes the above result in the special case of vanishing $\ell_{i}$. Since this deformation did not break conformal invariance, the energy goes as $1 / L$, since the only scale present is $L$. However, the charges are effectively enhanced by the factor $\sqrt{H}$. Notice that the $\gamma$ deformations have no effect in this regard, so long as the string is oriented purely in the radial direction.

\section{Deformations of Coulomb branch flows}

We would now like to investigate the effects of these deformations on the quark anti-quark potential for nonconformal field theories, in particular with regards to confinement. The natural place to begin such endeavors is to consider deformations of theories which already exhibit confinement. It turns out that for the Klebanov-Strassler [22] and Maldacena-Núñez 23. backgrounds, both of which exhibit confining IR phases, the quark anti-quark potential remains invariant under $\gamma$ deformations. From the last section, we might wonder if $\sigma$ deformations might enhance the scale of confinement. However, these backgrounds are not well-defined under $\sigma$ deformations [4, 12]. We do not consider these types of deformations of the Polchinski-Strassler solution [24] because they would break all of the R-symmetry and therefore not be supersymmetric. In addition, since it is a perturbative solution, we are not guaranteed that these deformations would maintain the regularity of the solution.

The quark anti-quark potential has been studied for the $\gamma$ deformed Coulomb branch of $\mathcal{N}=4$ super Yang Mills theory [18], where $\gamma$ deformations were found to effect the behavior only when the two $U(1)$ factors in the global gauge group corresponding to the quark and anti-quark are given different expectation values. We would like to consider $\sigma$ deformations of this theory. The supergravity dual of the deformed Coulomb branch flow can be written 
(with the metric expressed in the string frame) as [13]

$$
\begin{aligned}
d s^{2} & =\alpha^{\prime} \sqrt{H f} R^{2}\left[\frac{G}{g} s_{\alpha}^{2}\left(D \varphi_{1}+g L_{3} c_{\theta}^{2} D \varphi_{2}\right)^{2}+\frac{G}{4 h} D \varphi_{2}^{2}+\frac{r^{2}}{f R^{4}} d x_{\mu}^{2}+\frac{d r^{2}}{f r^{2} L_{1} L_{2} L_{3}}\right. \\
& \left.+k^{-1}\left[d \alpha+\frac{k}{4}\left(L_{2}-L_{3}\right) s_{2 \alpha} s_{2 \theta} d \theta\right]^{2}+k f^{-1} s_{\alpha}^{2} d \theta^{2}+\frac{9}{4} g h L_{1} L_{2} L_{3} s_{2 \alpha}^{2} s_{2 \theta}^{2} d \psi^{2}\right], \\
F_{(5)} & =d C_{(4)}+* d C_{(4)}, \quad C_{(4)}=\left(f^{-1} \frac{r^{4}}{R^{4}}-1\right) d^{4} x, \\
B & =\gamma \frac{R^{4} f s_{\alpha}^{2}}{4 g h} G D \varphi_{1} \wedge D \varphi_{2}-\sigma \mathcal{A}_{(2)}, \quad C^{(2)}=-\sigma \frac{R^{4} f s_{\alpha}^{2}}{4 g h} G D \varphi_{1} \wedge D \varphi_{2}-\gamma \mathcal{A}_{(2)}, \\
e^{2 \phi} & =H^{2} G, \quad \chi=\gamma \sigma \frac{R^{4} f s_{\alpha}^{2}}{4 g h H},
\end{aligned}
$$

where

$$
\begin{aligned}
d \mathcal{A}_{(2)} & =\frac{3}{4} R^{4} \frac{f^{2}}{r} s_{2 \alpha} s_{2 \theta} d \psi \wedge\left(\frac{L_{1} L_{2} L_{3}}{r^{2}} \partial_{r}\left(f^{-1} r^{4}\right) s_{\alpha}^{2} d \alpha \wedge d \theta+k f^{-1} \partial_{\alpha}\left(f^{-1}\right) s_{\alpha}^{2} d r \wedge d \theta\right. \\
& \left.+\left[\frac{L_{3}-L_{2}}{4} s_{2 \alpha} s_{2 \theta} \partial_{\alpha}\left(f^{-1}\right)+\frac{1}{k} \partial_{\theta}\left(f^{-1}\right)\right] d r \wedge\left[d \alpha+\frac{k}{4}\left(L_{2}-L_{3}\right) s_{2 \alpha} s_{2 \theta} d \theta\right]\right)
\end{aligned}
$$

and $D \varphi_{i}=d \varphi_{i}+A_{\psi}^{i} d \psi$ with the connection one-forms

$$
A_{\psi}^{1}=\left[L_{3} c_{\theta}^{2}\left(1-A_{\psi}^{2}\right)-L_{2} s_{\theta}^{2}\right] g, \quad A_{\psi}^{2}=\left(2 g L_{2} L_{3} s_{\alpha}^{2} s_{2 \theta}^{2}-4 L_{1} c_{\alpha}^{2}\right) h .
$$

The various functions are given by

$$
\begin{aligned}
& f^{-1}=\left(L_{1}^{-1} c_{\alpha}^{2}+L_{2}^{-1} s_{\alpha}^{2} s_{\theta}^{2}+L_{3}^{-1} s_{\alpha}^{2} c_{\theta}^{2}\right) L_{1} L_{2} L_{3}, \quad g^{-1}=L_{2} s_{\theta}^{2}+L_{3} c_{\theta}^{2}, \\
& h^{-1}=4 L_{1} c_{\alpha}^{2}+g L_{2} L_{3} s_{\alpha}^{2} s_{2 \theta}^{2}, \quad k^{-1}=L_{1} s_{\alpha}^{2}+g^{-1} c_{\alpha}^{2},
\end{aligned}
$$

and

$$
L_{i}=1+\frac{\ell_{i}^{2}}{r^{2}}
$$

The deformation functions are

$$
G^{-1}=1+\left(\hat{\gamma}^{2}+\hat{\sigma}^{2}\right) \frac{f}{g h} s_{\alpha}^{2}, \quad H=1+\hat{\sigma}^{2} \frac{f}{g h} s_{\alpha}^{2} .
$$

The deformations turn on the complex 3-form field strength, which supports D5 and NS5branes wrapped on a two-torus. As discussed in [4], in order for the 5-brane charges to be quantized, $\gamma$ and $\sigma$ must take on rational values. Also, since the dilaton is no longer constant, the corresponding super Yang-Mills coupling constant runs along the flow.

The deformed geometry is regular [13], with the exception of the singular shell of D3branes which may represent a phase transition at the associated scale of the RG flow [14. The conditions for the classical supergravity description to be valid include those for the 
marginal deformations of $A d S_{5} \times S^{5}$, and are given by (2.3). Even so, the curvature blows up as one approaches the singular shell of D3-branes which, from the point of view of the dual gauge theory, may correspond to a phase transition [14]. This is not a problem for us as long as the curvature is small at the minimal radius $r_{0}$ which is probed by string configurations. This is guaranteed if $r_{0}>>\ell_{i} / R^{2}$. All of these conditions are met for large enough $R$.

We will now consider inserting a probe D3-brane at large distance and consider the behavior of string configurations which end on this brane. For the purposes of Wilson loop calculations, only the metric is important. Since we will only consider static string configurations, antisymmetric tensor fields do not contribution to the string action. The symmetry in the $x_{i}$ directions allows us to choose coordinates so that the trajectory runs in the $x_{1} \equiv x$ direction with $x_{2}=x_{3}=0$. Also, since the metric is independent of $\varphi_{i}$ and $\psi$, taking them to be constant is automatically consistent with the corresponding equations of motion. With these simplifications, the string action is

$$
S=\frac{T}{2 \pi} \int d x \sqrt{H} \sqrt{\frac{r^{4}}{f R^{4}}+\frac{r^{\prime 2}}{f L_{1} L_{2} L_{3}}+\frac{r^{2}}{k} \alpha^{\prime 2}+\frac{r^{2} k}{f} s_{\alpha}^{2} \theta^{\prime 2}+\frac{r^{2}}{2}\left(L_{2}-L_{3}\right) s_{2 \alpha} s_{2 \theta} \alpha^{\prime} \theta^{\prime}}
$$

where' denotes a derivative with respect to $x$ and $T$ denotes the time interval. A simple solution to the equations of motion is given by $\alpha=\pi / 2, \theta=\pi / 4$ and $\ell_{2}=\ell_{3}$. This restricts us to the case with $S O(4) \times S O(2)$ symmetry. The trajectories are parallel to an axis that passes through the center of the D3-brane distribution. Notice that, for a spherical distribution of branes, all $\ell_{i}$ are equal and $H$ reduces the constant values of the previous section. Since the action does not explicitly depend on $x_{1}$, the solution satisfies

$$
\sqrt{\frac{H}{f}} \frac{r^{4}}{\sqrt{\frac{r^{4}}{R^{4}}+\frac{r^{\prime 2}}{L_{1} L_{2}^{2}}}}=\sqrt{\frac{H_{0}}{f_{0}}} r_{0}^{2} R^{2}
$$

where $H_{0} \equiv H\left(r_{0}\right), f_{0} \equiv f\left(r_{0}\right)$ and $r_{0}$ is the minimum value of $r$ that the string reaches. The solution is given by

$$
x=R^{2} \int_{r_{0}}^{r} \frac{d r}{r^{2} \sqrt{L_{1}} L_{2} \sqrt{\frac{f_{0} H r^{4}}{f H_{0} r_{0}^{4}}-1}},
$$

where $r_{0}$ is determined by $L=2 x(r \rightarrow \infty)$.

Figure 2 shows string configurations for $\ell_{1}=0, \ell_{2}=\ell_{3}=10$. The plots for $\hat{\sigma}=1$ (black), 2 (red) and 3 (blue) serve to demonstrate that, at a given $L$, the $\sigma$ deformations increase the energy scale $(r)$ probed by a string. One condition in order for the $\sigma$ deformations to induce a phase transition to a confining phase is that the energy scale must be enhanced all the more for large $L$. 


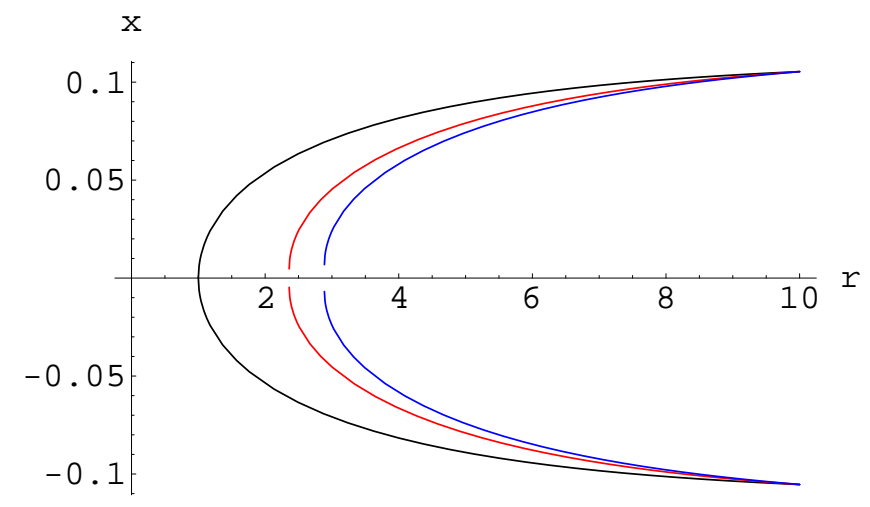

Figure 2: String configuration for $\ell_{1}=0, \ell_{2}=\ell_{3}=10$ and $\hat{\sigma}=1$ (black), 2 (red) and 3 (blue). For a given $L$, the $\sigma$ deformations increase the energy scale probed by a string.

The regularized energy of the string configuration is

$$
E=\frac{1}{\pi} \int_{r_{0}}^{\infty} d r\left[\sqrt{\frac{f_{0}}{H_{0}}} \frac{H r^{2}}{f r_{0}^{2} \sqrt{L_{1}} L_{2} \sqrt{\frac{f_{0} H r^{4}}{f H_{0} r_{0}^{4}}-1}}-\sqrt{1+\hat{\sigma}^{2}}\right]-\frac{1}{\pi} \int_{0}^{r_{0}} d r \sqrt{1+\hat{\sigma}^{2}},
$$

where the infinite contribution from the W-bosons has been subtracted. $L$ and $E$ can be written in terms of complete elliptic integrals for two separate cases [25].

The first case is given by

$$
\begin{aligned}
& L=4 R^{2}\left(1+\hat{\sigma}^{2}\right) \beta_{1} \sqrt{\left(r_{0}^{2}+\ell_{2}^{2}\right) \alpha_{1}}\left[\Pi\left(\alpha_{1}, \sqrt{\alpha_{2}}\right)-K\left(\sqrt{\alpha_{2}}\right)\right], \\
& E=\frac{\sqrt{\beta_{2}}}{2 \pi}\left[\frac{\alpha_{1}}{\beta_{2}} K\left(\sqrt{\alpha_{2}}\right)-\frac{\left(1+\hat{\sigma}^{2}\right)\left(r_{0}^{2}+\ell_{1}^{2}\right)}{1-\alpha_{2}} E\left(\sqrt{\alpha_{2}}\right)\right] .
\end{aligned}
$$

where ${ }^{5}$

$$
\alpha_{i} \equiv\left[r_{0}^{2}+\ell_{i}^{2}+\hat{\sigma}^{2}\left(r_{0}^{2}+2 \ell_{2}^{2}-\ell_{3-i}^{2}\right)\right] \beta_{2}, \quad \beta_{i}^{-1} \equiv 2 r_{0}^{2}+\ell_{1}^{2}+\ell_{i}^{2}+2 \hat{\sigma}^{2}\left(r_{0}^{2}+\ell_{2}^{2}\right) .
$$

This case applies when either $r_{0}^{2} \geq \ell_{1}^{2}-2 \ell_{2}^{2}$ for any $\hat{\sigma}$, or else $r_{0}^{2}<\ell_{1}^{2}-2 \ell_{2}^{2}$ and $\hat{\sigma}^{2}<\frac{r_{0}^{2}+\ell_{2}^{2}}{\ell_{1}^{2}-2 \ell_{2}^{2}-r_{0}^{2}}$. For equal $\ell_{i}, L$ becomes independent of $\hat{\sigma}$ and reduces to that of the conformal case considered in [16]. For vanishing $\hat{\sigma}$ this reduces to the undeformed Coulomb branch, particular cases of which have been considered in [17].

Figures 3 and 4 are parametric plots of the distance between the quark and the antiquark $L$ versus the quark anti-quark potential $E$ using (3.11) for case 1. The effect of the

\footnotetext{
${ }^{5}$ We denote $K, E$, and $\Pi$ for the complete elliptic integrals of the first, second and third kind, respectively.
} 
$\sigma$ deformations depends on which part of the Coulomb branch we are probing, as well as the trajectories of the probe strings. For trajectories which are orthogonal to a uniform distribution of D3-branes on a two-dimensional disk of radius $\ell_{1}\left(\ell_{1} \neq 0, \ell_{2}=\ell_{3}=0\right)$, we find that the $\sigma$ deformations enhance the attractive Coulombic force between the quark and anti-quark. This force vanishes at asymptotically large distance ${ }^{6}$. This is shown in Figure 3 for $\ell_{1}=1$ and $\hat{\sigma}=0,10$ and 20. We have added $\sigma$ dependent constants to the energy (since energy is only defined up to an additive constant) in order to render it zero when the quark anti-quark distance gets very large. We have set $R$ to unity for convenience, since it simply has the effect of rescaling $\hat{\sigma}$ and $L$.

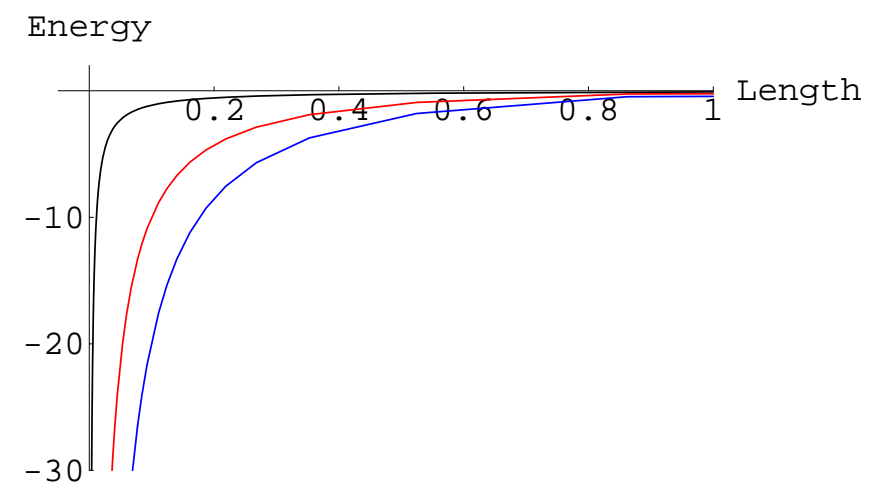

Figure 3: Quark anti-quark potential using (3.11) for $\ell_{1}=1, \ell_{2}=\ell_{3}=0$ and $\hat{\sigma}=0$ (black), 10 (red) and 20 (blue). In this part of the Coulomb branch, the $\sigma$ deformations simply enhance the Coulombic force.

For a uniform distribution of D3-branes on a three-dimensional spherical $\operatorname{shell}^{7}\left(\ell_{1}=0\right.$ and $\ell_{2}=\ell_{3} \neq 0$ ), the sigma deformations actually induce a phase transition from a Coulombic phase for small distance to a linearly confining phase as the distance becomes larger. This is shown in Figure 4 for the case of $\ell_{1}=0, \ell_{2}=\ell_{3}=10$ and $\hat{\sigma}=1,2$ and 3. For small distances between the quarks and anti-quarks, they still experience Coulombic attraction, as in the case of the conformal fixed point in the UV limit. As the distance increases, this transitions to a regime of linear confinement, where the scale of confinement increases with $\hat{\sigma}$. This is very similar to what happens for certain string trajectories in parts of the moduli space of the undeformed theory which exhibit a confinement phase, such as case II in [17] as shown in

\footnotetext{
${ }^{6}$ Our results indicate that there is complete screening for large enough $L$, as was found for these trajectories in the undeformed backgrounds [17. However, this occurs only for strings which touch the D3-brane distribution and is simply a remnant of the continuous approximation of the distribution.

${ }^{7}$ This can be obtained from the disk distribution by taking $\ell_{1}^{2} \rightarrow-\ell_{1}^{2}$ and $r \geq \ell_{1}$, as in [18.
} 


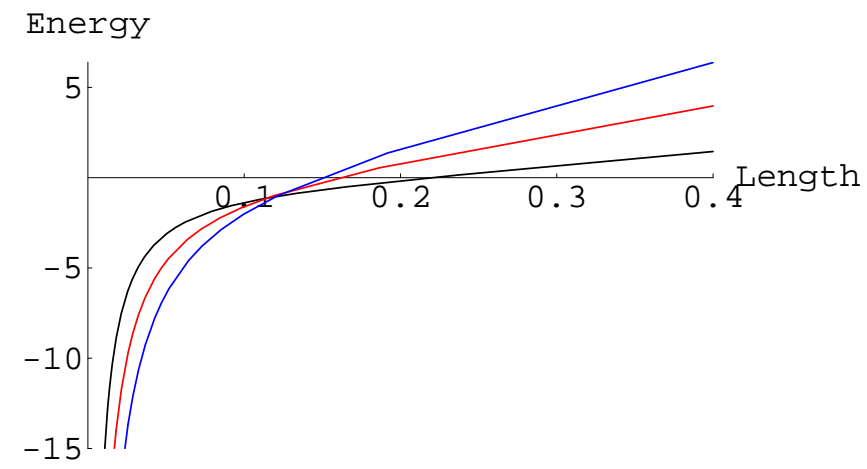

Figure 4: Quark anti-quark potential using (3.11) for $\ell_{1}=0, \ell_{2}=\ell_{3}=10$ and $\hat{\sigma}=1$ (black), 2 (red) and 3 (blue). $R$ has been set to unity. For small distance, the force is Coulombic. As the distance increases in this part of the Coulomb branch, the $\sigma$ deformations induce a transition to a linearly confining phase. The scale of confinement increases with $\sigma$.

Figure 5 of that paper. However, in the limit of vanishing $\sigma$, our trajectory matches that of case I in [17, for which there was no confining behavior.

In order to see the behavior for large length $L$, consider (3.11) as $\alpha_{2} \rightarrow 1$, which implies that $r_{0}$ and $\ell_{1}$ are small. In this approximation, we get the simple analytical result

$$
E=\frac{\hat{\sigma} \ell_{2}^{2}}{2 \pi R^{2}} L
$$

This result can be obtained either by expanding the complete elliptic integrals around $\alpha_{2}=1$, or equivalently by assuming that the dominant contribution arises from the region near $r=r_{0}$. As expected, for nonzero $\hat{\sigma}$ we have linear confinement at large distance. Note that the scale of confinement is independent of the coupling constant $g_{Y M}$ in this limit. In the cases considered in Figure 4, the typical length scale at which the Coulombic behavior transitions to linear confinement is on the order of $.2 R^{2} / \ell_{2}$.

For our deformed solution (3.1), similar behavior can also be found for a uniform distribution of D3-branes on a particular family of five-dimensional ellipsoids (all $\ell_{i}$ nonvanishing and $\left.\ell_{2}=\ell_{3}\right)$. For the more general examples with $S O(2) \times S O(2) \times S O(2)$ symmetry $\left(\ell_{2} \neq \ell_{3}\right)$, there are no longer any purely radial trajectories. Nevertheless, we expect to find that these modes also undergo transitions to confining phases. For nonradial trajectories, $\gamma$ deformations also have an effect. Cases in which there is linear as well as logarithmic confining behavior were studied in [18].

The second case for which $E$ and $L$ can be written in terms of complete elliptic integrals 
25 is

$$
\begin{aligned}
L & =\frac{R^{2}}{\left(\ell_{1}^{2}-\ell_{2}^{2}\right)} \sqrt{\frac{r_{0}^{2}+\ell_{2}^{2}}{2 \beta_{1}\left(1+\hat{\sigma}^{2}\right)\left(r_{0}^{2}+\ell_{1}^{2}\right)}}\left[\Pi\left(\frac{\ell_{1}^{2}-\ell_{2}^{2}}{r_{0}^{2}+\ell_{1}^{2}}, \sqrt{q}\right)-K(\sqrt{q})\right], \\
E & =\frac{1}{2 \pi \beta_{2} \sqrt{\left(1+\hat{\sigma}^{2}\right)\left(r_{0}^{2}+\ell_{1}^{2}\right)}}\left[\alpha_{1} K(\sqrt{q})-\frac{1}{(1-q)} E(\sqrt{q})\right],
\end{aligned}
$$

where

$$
q \equiv \frac{\hat{\sigma}^{2} \ell_{1}^{2}-\left(1+2 \hat{\sigma}^{2}\right) \ell_{2}^{2}-\left(1+\hat{\sigma}^{2}\right) r_{0}^{2}}{\left(1+\hat{\sigma}^{2}\right)\left(r_{0}^{2}+\ell_{1}^{2}\right)} .
$$

This case applies when $r_{0}^{2}<\ell_{1}^{2}-2 \ell_{2}^{2}$ and $\hat{\sigma}^{2}>\frac{r_{0}^{2}+\ell_{2}^{2}}{\ell_{1}^{2}-2 \ell_{2}^{2}-r_{0}^{2}}$. Since $\hat{\sigma}$ is nonvanishing for this case, there is no limit in which it reduces to undeformed results. We have found similar behavior as shown in Figures 3 and 4 for the various values of $\ell_{i}$ and $\sigma$.

\section{Discussion}

We have considered the gravity dual of deformations of the Coulomb branch of $\mathcal{N}=4$ super Yang-Mills theory. In particular, for $\sigma$ deformations we have used Wilson loops to probe the geometry in cases which preserve $S O(4) \times S O(2)$ symmetry. In certain parts of the moduli space, we have found that $\sigma$ deformations induce a transition from Coulombic attraction between quarks and anti-quarks to linear confinement.

We have already noted that the supergravity description breaks down for string configurations which get too close to the singular shell of D3-branes. In this region, one can no longer approximate the brane distribution as being continuous. This description must be replaced by discretely-spaced stacks of D3-branes. We can now consider a string getting close to a particular stack which has a large number $n$ of D3-branes, where $1<<n<<N$. For the cases considered in Figure 3, for which the Coulombic behavior persists after the deformations, there is partial screening of charges since $R^{4}$ is effectively reduced by a factor of $n / N$ [18. For cases in which there is linear confinement, as presented in Figure 4, we find that the confinement is actually occurring at an intermediate energy scale of the theory. In both very high and low energy scales, there is Coulombic behavior. There is partial charge screening in the deep IR region relative to the UV limit.

The concavity condition on the potential of a heavy quark anti-quark pair is given by

$$
\frac{d E}{d L}>0, \quad \frac{d^{2} E}{d L^{2}} \leq 0
$$

It can be seen directly from Figures 3 and 4 that these conditions are obeyed. The first condition implies that the force between the quark and anti-quark is always attractive. Although 
this is obvious from QCD, this is not obvious from the viewpoint of the string theory a priori. The second condition implies that the force does not increase with $L$. The concavity condition is sometimes not satisfied when the string configuration is too close to the D3-brane shell, since then the continuous approximation of the brane distribution breaks down [17].

Although the $\beta$ deformations can have a dramatic effect on the behavior of radial string configurations, the minimally-coupled massless scalar field equation for purely radial modes remains unchanged. This seems to indicate that the quarks and glueballs in the deformed theory are associated with two independent confinement scales ${ }^{8}$. On the other hand, scalar modes with angular dependence are affected by $\beta$ deformations. This was discussed for the case of $\gamma$ deformations in [18, where it was found that the wave equation can be written as the Heun differential equation, which is related to the $\mathrm{BC}_{1}$ Inozemtsev system by way of a non-standard trigonometric limit. Since this is an integrable model, the Bethe ansatz method can be used to find solutions [18]. For the full $\beta$ deformations, the $H$ factors from the background metric in (3.1) cancel out of the wave equation, regardless of angular dependence. From $G$ in (3.6), the wave equation has the same form as that for $\sigma=0$ with the replacement $\gamma \rightarrow \beta$ and is therefore integrable.

In the case of $\gamma$ deformations, it has been explicitly shown that the deformed supergravity solution preserves $\mathcal{N}=1$ supersymmetry [26]. We expect that the same is true for $\sigma$ deformations, since the direction corresponding to the $U(1)$ R-symmetry is not involved in the deformation procedure. However, it would be nice to show this from the Killing spinor equations.

We have restricted ourselves to only purely radially oriented string configurations. One could also consider strings whose endpoints lie at different angles in the internal space. This would correspond to giving different expectation values to the two $U(1)$ factors in the global gauge group corresponding to the quark and the anti-quark [16]. Such configurations have already been considered for the supergravity backgrounds describing the undeformed Coulomb branch of $\mathcal{N}=4$ Yang Mills in [17], as well as for the $\gamma$ deformed backgrounds in [18]. However, one of the main points of this paper is that $\sigma$ deformations, as opposed to $\gamma$ deformations, can have a radical effect on the behavior of purely radial string configurations.

It would also be interesting to consider how these $\sigma$ deformations change the confining characteristics and phase structure of finite temperature field theories described by nonextremal rotating D3-branes.

Finally, in this paper we have only considered external quarks. It would certainly be nice to consider dynamical quarks through the addition of D7-branes.

\footnotetext{
${ }^{8}$ We are grateful to Leopoldo Pando Zayas for bringing up this possibility
} 


\section{Acknowledgments}

We would like to thank Philip Argyres, Sangmin Lee, Hai Lin, Hong Lü, Oleg Lunin, Carlos Núñez, Chris Pope, Konstadinos Sfetsos and Rohana Wijewardhana for useful discussions. We are grateful to comments made by Konstadinos Sfetsos regarding the earlier version of this paper. We thank the Institute for Advanced Study for hospitality during the early stages of this work. C.A. thanks the Korea Institute for Advanced Study (KIAS) where this work was undertaken and his work was supported by grant No. R01-2006-000-10965-0 from the Basic Research Program of the Korea Science \& Engineering Foundation. The work of J.F.V.P. is supported by DOE grant DOE-FG02-84ER-40153.

\section{References}

[1] J.M. Maldacena, The large $N$ limit of superconformal field theories and supergravity, Adv. Theor. Math. Phys. 2 (1998) 231; Int. J. Theor. Phys. 38, 1113 (1999), hep-th/9711200.

[2] S. S. Gubser, I. R. Klebanov and A. M. Polyakov, Gauge theory correlators from noncritical string theory, Phys. Lett. B 428, 105 (1998), hep-th/9802109.

[3] E. Witten, Anti-de Sitter space and holography, Adv. Theor. Math. Phys. 2, 253 (1998), hep-th/9802150

[4] O. Lunin and J. Maldacena, Deforming field theories with $U(1) \times U(1)$ global symmetry and their gravity duals, JHEP 0505, 033 (2005), hep-th/0502086.

[5] R. G. Leigh and M. J. Strassler, Exactly marginal operators and duality in fourdimensional $N=1$ supersymmetric gauge theory, Nucl. Phys. B 447, 95 (1995), hep-th/9503121

[6] C. Ahn and J. F. Vázquez-Poritz, Marginal deformations with $U(1)^{3}$ global symmetry, JHEP 0507, 032 (2005), hep-th/0505168.

[7] J. P. Gauntlett, D. Martelli, J. Sparks and D. Waldram, Supersymmetric AdS(5) solutions of M-theory, Class. Quant. Grav. 21, 4335 (2004), hep-th/0402153.

[8] J. P. Gauntlett, D. Martelli, J. Sparks and D. Waldram, Sasaki-Einstein metrics on S(2) $x$ S(3), Adv. Theor. Math. Phys. 8, 711 (2004), hep-th/0403002.

[9] M. Cvetič, H. Lü, D. N. Page and C. N. Pope, New Einstein-Sasaki Spaces in Five and Higher Dimensions, Phys. Rev. Lett. 95, 071101 (2005), hep-th/0504225. 
[10] D. Martelli and J. Sparks, Toric Sasaki-Einstein metrics on S(2) $x$ S(3), Phys. Lett. B621, 208 (2005), hep-th/0505027.

[11] J. P. Gauntlett, S. Lee, T. Mateos and D. Waldram, Marginal deformations of field theories with $A d S_{4}$ duals, JHEP 0508, 030 (2005), hep-th/0505207.

[12] U. Gursoy and C. Núñez, Dipole deformations of N=1 SYM and supergravity backgrounds with $U(1) \times U(1)$ global symmetry, Nucl. Phys. B725, 45 (2005), hep-th/0505100.

[13] C. Ahn and J.F. Vázquez-Poritz, Deformations of flows from type IIB supergravity, to be published in Class. Quant. Grav., hep-th/0508075.

[14] P. Kraus, F. Larsen and S.P. Trivedi, The Coulomb branch of gauge theory from rotating branes, JHEP 9903, 003 (1999), hep-th/9811120.

[15] S. Rey and J. Yee, Macroscopic strings as heavy quarks in large $N$ gauge theory and anti-de Sitter supergravity, Eur. Phys. J. C22, 379 (2001), hep-th/9803001.

[16] J.M. Maldacena, Wilson loops in large N field theories, Phys. Rev. Lett. 80, 4859 (1998), hep-th/9803002.

[17] A. Brandhuber and K. Sfetsos, Wilson loops from multicentre and rotating branes, mass gaps and phase structure in gauge theories, Adv. Theor. Math. Phys. 3, 851 (1999), hep-th/9906201.

[18] R. Hernandez, K. Sfetsos and D. Zoakos, Gravity duals for the Coulomb branch of marginally deformed $N=4$ Yang-Mills, hep-th/0510132.

[19] N. Dorey, S-duality, deconstruction and confinement for a marginal deformation of $\mathcal{N}=4$ SUSY Yang-Mills, JHEP 0408, 043 (2004), hep-th/0310117.

[20] N. Dorey, A new deconstruction of little string theory, JHEP 0407, 016 (2004), hep-th/0406104.

[21] N. Dorey and T.J. Hollowood, On the Coulomb branch of a marginal deformation of $\mathcal{N}=4$ SUSY Yang-Mills, JHEP 0506, 036 (2005), hep-th/0411163

[22] I.R. Klebanov and M.J. Strassler, Supergravity and a confining gauge theory: duality cascades and $\chi S B$-resolution of naked singularities, JHEP 0008 (2000) 052, hep-th/0007191.

[23] J.M. Maldacena and C. Núñez, Towards the large $N$ limit of pure $N=1$ super Yang Mills, Phys. Rev. Lett. 86 (2001) 588, hep-th/0008001. 
[24] J. Polchinski and M.J. Strassler, The string dual of a confining four-dimensional gauge theory, hep-th/0003136.

[25] I.S. Gradshteyn and I.M. Ryzhik, Table of integrals, series and products, Sixth Edition, Academic Press, New York, 2000.

[26] R. Hernandez, K. Sfetsos and D. Zoakos, On supersymmetry and other properties of a class of marginally deformed backgrounds, Proceedings of the RTN Corfu workshop in September 2005, hep-th/0512158 